\title{
Análisis de los determinantes de la demanda de carne de pollo en el Centro-Este de México, 1996-2018
}

\author{
Analysis of demand poultry meat determinants in Central-Eastern Mexico, 1996-2018 \\ Samuel Rebollar-Rebollar ${ }^{1}$, Eulogio Rebollar-Rebollar ${ }^{* *}$, Juvencio Hernández-Martínez²
}

\begin{abstract}
${ }^{1}$ Centro Universitario UAEM-Temascaltepec, Universidad Autónoma del Estado de México, (UAEM), Carretera Toluca-Tejupilco Km. 67.5, Colonia Barrio de Santiago s/n, 51300, Temascaltepec, Estado de México, México.

${ }^{2}$ Centro Universitario UAEM-Texcoco, Universidad Autónoma del Estado de México, (UAEM), Av. Jardín Zumpango s/n, Fracc. El Tejocote, 56259, Texcoco, Estado de México, México.
\end{abstract}

*Autor para correspondencia: rebollar55@hotmail.com

Fecha de recepción

12 de enero de 2020

Fecha de aceptación

15 de marzo de 2020

Disponible en línea

2 de abril de 2020

Este es un artículo en acceso abierto que se distribuye de acuerdo a los términos de la licencia Creative Commons

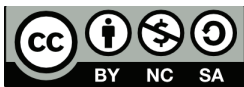

ReconocimientoNoComercial-CompartirIgual 4.0 Internacional

\section{RESUMEN}

El objetivo del presente estudio fue analizar los determinantes de la demanda de carne de pollo en la región Centro-Este de México, como los precios de las carnes de pollo, cerdo y bovino, así como el ingreso y la población. Se probaron dos modelos econométricos; el que tuvo el mejor ajuste fue el de regresión lineal múltiple, conforme a las pruebas estadísticas de la R-cuadrada ajustada, error estándar, t de Student, así como de las hipótesis económicas. El precio del producto fue inelástico (-0.21) sobre la demanda de pollo; el ingreso del consumidor y el precio de la carne de bovino tuvieron respuesta positiva e inelástica (0.09 y 0.16); asimismo, el precio de la carne de cerdo fue negativa e inelástica (-0.16); en tanto que, respecto a la población, la respuesta fue directa y elástica (2.46), e inelástica con el ingreso. La carne de bovino resultó el sustituto más cercano, mientras que la carne de cerdo fue un bien complementario.

PALABRAS CLAVE

consumo, aves de corral, elasticidades

\section{ABSTRACT}

The objective of this study was to analyze the demand poultry meat determinants in the Central-Eastern region of Mexico, such as the prices of poultry, pork and beef, income and population. Two econometric models were tested; the model that had the best fit was the multiple linear regression, according to the statistical tests, adjusted R-square, standard error, Student's t-test, as well as the economic hypotheses. Price of the product was inelastic $(-0.21)$ on the demand for poultry; the consumer income and beef price had a positive and inelastic response $(0.09$ and 0.16$)$; the price of pork was negative and inelastic $(-0.16)$; while the population the response was direct and elastic (2.46), and inelastic with income. Bovine meat is the closest substitute, pork was a complementary good.

KEYWORDS

consumption, poultry, elasticities 


\section{INTRODUCCIÓN}

La avicultura comercial mexicana se reconoce como una de las actividades pecuarias con mayor realce, con relación a los bovinos y porcinos, lo cual se justifica por el crecimiento permanente tanto en producción como en consumo (SIAP 2020), pero también porque tal actividad está mejor integrada (Medina et al. 2012). En 2018, cifras oficiales mexicanas permitieron verificar que el volumen total producido de todas las carnes pecuarias de interés económico sumó 6.94 millones de toneladas. De ese total, las aves de carne contribuyeron con $47.98 \%$, equivalente a 3.33 millones de toneladas; los bovinos, con $28.53 \%$; los porcinos, con $21.61 \%$, y el resto de las carnes lo hicieron con $1.88 \%$; las entidades federativas de importancia fueron Jalisco, Veracruz, Querétaro, Durango, Aguascalientes y Guanajuato, quienes colaboraron con $60.21 \%$ (SIAP 2020).

Para el periodo del presente estudio (1996 a 2018), el consumo mexicano de carne de pollo aumentó a una tasa anual promedio cercana a 5.13\%. Así, mientras que en 1996 la población consumió 1.39 millones de toneladas, en 2018 esta cifra llegó a 4.10. El aumento obedece a la disponibilidad per cápita, que creció $4.33 \%$; es decir, de $14 \mathrm{~kg} /$ habitante en 1996, pasó a 33.1 en 2018 (SIAP 2020).

Del volumen total nacional consumido de carne de pollo en 2018, la producción participó con $86.60 \%$ y las compras externas lo hicieron en $13.40 \%$ importaciones. Cabe mencionar que, en México, y durante ese periodo, las exportaciones de carne de pollo no impactaron el consumo nacional (UNA 2019).

En ese mismo periodo, la dinámica creciente del consumo de esta carne se debió a la evolución del ingreso real per cápita del consumidor (3.39\%) (INEGI 2019), al crecimiento nacional del número de habitantes (1.24\%) (CONAPO 2019), y a la disminución del precio real de la carne de pollo, equivalente a una tasa media de crecimiento anual de $-0.16 \%$ (26.89 pesos mexicanos por $\mathrm{kg}$ en 1996 a 25.01 en 2018). Aunado a esto, hubo un cambio de preferencias por parte del consumidor en favor de esta carne, por motivos vinculados con la cantidad de integrantes de la familia y el ingreso monetario, los cuales beneficiaron positivamente las posibilidades de consumo de carne (Tellez et al. 2016).

Para 2018, el consumo mexicano de carne de pollo se centró, principalmente, en la región Centro-Este (CE) del país, que abarca la Ciudad de México (CDMX), así como Hidalgo, el Estado de México, Morelos, Puebla, Querétaro y Tlaxcala (Rebollar-Rebollar et al. 2019), con un volumen oficial registrado de $33.17 \%$ respecto al total nacional, es decir, 1.36 millones de toneladas, lo que la ubica como la región consumidora principal de carne de pollo en México (SIAP 2020).

Por lo anterior, el objetivo de este trabajo consistió en evaluar el efecto de los determinantes de la demanda de carne de pollo en la región CE de México, con base en sus elasticidades para el periodo 1996-2018. La hipótesis central afirma que la demanda de carne de pollo en la región CE de México responde de forma inversa al comportamiento del precio real del producto, se explica de forma directa por el ingreso real per cápita y por el precio real de la carne bovina, inversa al precio real de la carne de cerdo y directa por la población.

\section{Materiales Y MÉTOdos}

Para analizar el efecto de los determinantes de la demanda de carne de pollo en canal en la región Centro-Este de México (CE), se utilizó información secundaria disponible de la serie 1996-2018.

Primero, se consideró la regionalización de Bassols (1992), en la que la región Centro-Este de México incorpora las siguientes entidades federativas: Ciudad de México (CDMX), Estado de México, Hidalgo, Querétaro, Tlaxcala, Puebla y Morelos.

En este estudio y para esta región del país, la variable explicada fue la demanda de carne de pollo en función de las predeterminadas: precio de la carne de pollo en canal, Producto Interno Bruto per cápita como medida de aproximación al ingreso del consumidor, precio de la carne de bovino y porcino en canal, y la población, como consumidores de esa carne.

Se utilizó información estadística generada por fuentes secundarias oficiales de México, como CONAPO (2019), SIAP (2020), FIRA (2019), SNIIM (2019). Todas las variables monetarias relacionadas a las variables explicativas se deflactaron con la utilización del Índice Nacional de Precios al Consumidor (INPC), base 2012 (INEGI, 2018).

Para determinar el modelo mejor ajustado a la masa de datos disponible, se decidió correr dos modelos econométricos, con el propósito de observar la significancia estadística. Así, el primero de ellos fue multiplicativo y el segundo de regresión lineal múltiple (RLM). En los dos modelos se consideraron los determinantes del consumo de este subproducto pecuario en esa región de México; por ello, con base en Salvatore (2009) y Vázquez y Martínez (2015), se afirma que la demanda de cualquier producto en el 
mercado se da en función del precio del producto (bien o artículo), ingreso del comprador, precio de bienes sustitutos y complementarios, el gusto, la población y otros factores.

Luego de efectuarse las corridas con el modelo multiplicativo (con su transformación lineal mediante logaritmo), se observó que, en la salida de resultados, el signo de los coeficientes de las variables fijas fue contrario a la teoría microeconómica, el error estándar asociado a cada estimador era demasiado grande, las $\mathrm{t}$-calculadas fueron menores a la unidad, por lo que se dedujo que los estimadores no fueron estadísticamente significativos; ni con tres periodos de retraso de las predeterminadas, hubo significancia en el modelo multiplicativo. En consecuencia, se optó por utilizar el modelo de regresión lineal múltiple (MRLM) y, después de llevar a cabo tantas cuantas corridas fue necesario, se observó que el ajuste de la masa de datos fue el esperado según la teoría microeconómica.

Por tanto, para estimar el valor de los parámetros en el modelo lineal asociado a la función de demanda, se utilizó el método de Mínimos Cuadrados Ordinarios (MCO) (Gujarati y Porter 2010), con el paquete estadístico SAS (2003).

La congruencia estadística del modelo lineal de demanda estimado (Wooldridge 2009), se determinó con el coeficiente de determinación $\mathrm{R}^{2}$-ajustado, la significancia estadística se efectuó mediante la prueba $\mathrm{F}$ (F-calculada) y la significancia estadística individual de cada coeficiente con la t de Student o la razón de t.

Con referencia en Wooldridge (2009), la significancia económica se hizo al considerar los signos y la magnitud de los coeficientes de las variables de la función de demanda (las variables independientes, regresadas, explicativas o predeterminadas), y su interpretación se realizó con fundamentos de la teoría microeconómica, mediante las elasticidades; es decir, con base en la ley de la demanda, la relación entre el volumen consumido de carne de pollo en canal en el periodo -aproximado por el consumo regional aparente (toneladas) - y el precio de la carne de pollo en canal (dado en pesos mexicanos por kilogramo de carne) debe ser inversa; mientras que con el Producto Interno Bruto real per cápita regional en el promedio del periodo (dado en miles de pesos mexicanos por persona) como una variable de aproximación al ingreso nacional disponible per cápita (Gujarati y Porter 2010), precio de la carne de bovino (expresada en pesos mexicanos por kilogramo), precio de la carne de cerdo y población (millones de habitantes), la relación debe ser directa, a menos que se obtenga lo contrario, en algunas de las explicativas.
Asimismo, se determinaron las elasticidades económicas por cada determinante de la demanda de carne de pollo en la región CE de México, y se evaluaron al considerar el signo y magnitud de sus coeficientes, mismos que interpretaron en términos de la teoría microeconómica de la demanda (Gujarati y Porter 2010; Nicholson y Snyder 2015; Parkin y Loría 2015).

El modelo estadístico que se utilizó para la demanda de carne de pollo en la región CE de México, 1996-2018, fue:

$\mathrm{DCP}_{\mathrm{t}}=\beta 11+\beta 12 \mathrm{PCPR}_{\mathrm{t}-1}+\beta 13 \mathrm{PIBRP}_{\mathrm{t}}+\beta 14 \mathrm{PRCB}_{\mathrm{t}-2}+$ $\beta 15 \mathrm{PRCC}_{\mathrm{t}-3}+\beta 16 \mathrm{POB}_{\mathrm{t}}+£_{\mathrm{t}}$

Donde, la variable dependiente $\mathrm{DCP}_{t}$ se refiere al volumen de demanda de carne de pollo en canal en el periodo actual, aproximado por el consumo aparente de la región CE (cifras dadas en miles de toneladas); $\mathrm{PCPR}_{\mathrm{t}}$ expresa el precio real promedio ponderado de carne de pollo en canal en el periodo actual (cifras en pesos mexicanos por kilogramo de carne) en la región $\mathrm{CE}$, rezagado un periodo; PIBRP: Producto Interno Bruto real per cápita de la región $C E$ en el periodo actual (cifras en miles de pesos mexicanos por persona), como una variable de aproximación al ingreso nacional disponible per cápita (Gujarati y Porter 2010; Vázquez y Martínez 2015); PRCB $_{t}$ : precio real promedio ponderado en la región $\mathrm{CE}$ de la carne de bovino (cifras en pesos mexicanos por kilogramo de carne), como producto sustituto, rezagado dos periodos; $\mathrm{PRCC}_{\mathrm{t}}$ fue el precio real promedio ponderado en la región CE de la carne de cerdo (cifras en pesos mexicanos por kilogramo de carne), como producto complementario, con rezago de tres periodos; $\mathrm{POB}_{\mathrm{t}}$ : población de la región $\mathrm{CE}$ en el periodo actual (cifras en millones de habitantes).

El consumo aparente de la región CE de México se determinó a partir de la estimación del consumo nacional, como una variable de aproximación a la demanda regional de este producto, definida por producción, más importaciones, menos exportaciones. Después, se multiplicó por el número de habitantes de la región CE de México el año determinado de la serie de tiempo considerada.

En consideración de la teoría microeconómica, los determinantes de la demanda que se incluyeron en el modelo fueron: precio real promedio de la carne de pollo en canal $\left(\mathrm{PRCP}_{\mathrm{t}}\right)$ de la región $\mathrm{CE}$, producto interno bruto real per cápita regional (CE) (PIBRP t $_{\mathrm{t}}$ de la de la región $\mathrm{CE}$, precio real de la carne de bovino como producto sustituto $\left(\mathrm{PRCB}_{\mathrm{t}}\right)$ de la región 
$\mathrm{CE}$, precio real de la carne de cerdo como producto sustituto (PRCCR $)$ de la región CE y la población (en número de personas) de la región $\mathrm{CE}\left(\mathrm{POB}_{\mathrm{t}}\right)$, todas las variables en el periodo actual. El PIBRP y la POB se incluyeron debido a que son variables que explican la demanda interna de alimentos en México (SAGARPA 2019).

Para determinar el valor de la elasticidad de la demanda con relación a cada una de las variables explicativas o predeterminadas, se multiplicó el coeficiente de la derivada parcial del modelo estimado por el valor promedio observado de la serie de tiempo de cada una de las variables independientes respecto de las cantidades demandadas (Ramírez-González et al. 2003; Cruz-Jiménez et al. 2016; Puebla et al. 2018). Asimismo, debido a que las funciones lineales de demanda tienen una elasticidad variable a través de su rango de estimación, ésta se obtuvo para el promedio del periodo analizado (Wooldridge 2009; Vázquez y Martínez 2015); así, se procedió a cuantificar los efectos establecidos en las relaciones funcionales del modelo de demanda de carne de pollo en canal.

\section{RESULTADOS Y DISCUSIóN}

El modelo mejor ajustado para la demanda de carne de pollo en canal en la región Centro-Este (CE) de México, periodo 1996-2018, fue el de regresión lineal múltiple:

$\mathrm{DCP}=-1,717.31-7.60 \mathrm{PCPR}+0.86 \mathrm{PIBRP}+4.22 \mathrm{PRCB}$ -6.00 PRCC + 76.20 POB

En este modelo, no hubo variables omitidas ni errores de especificación que pudiesen causar sesgo. $\mathrm{Al}$ ajustar la regresión de la DCP sobre las cinco variables fijas, los resultados que se obtuvieron fueron acordes a la teoría microeconómica. Sin embargo, al llevar a cabo las corridas omitiendo tanto la variable PRCB como la PRCC, el modelo arrojaba resultados contrarios a lo esperado; de ahí que la inclusión de los cinco regresores sobre la DCP generara los mejores resultados esperados.

Para conocer el valor del consumo de carne de pollo de la región $\mathrm{CE}$, se consideró información oficial del promedio de todo el periodo en cada una de las variables predeterminadas: $\mathrm{PCPR}=36.03 \$ / \mathrm{kg}$; PIBRP $=144.64$ miles de pesos; $\mathrm{PRCB}=49.67 \$ / \mathrm{kg} ; \mathrm{PRCC}=$ $34.26 \$ / \mathrm{kg}$; POB $=41.15$ millones de habitantes; con lo que la estimación de la DCP para la región CE fue de 1,272.78 miles de toneladas. Los resultados de los parámetros estimados (llamados estimadores) en el modelo de regresión, más la información de las variables explicativas, se utilizaron para determinar la elasticidad microeconómica de cada variable independiente o predeterminada del modelo.

\section{AnÁlisis ESTADÍstico}

La significancia global del modelo fue evidente, pues las variables explicativas relevantes que se incluyeron permitieron obtener una F-calculada $(\mathrm{Fc})$ de 406.46 a $95 \%$ de confiabilidad $(\mathrm{P}<0.0001)$ (cuadro 1). El coeficiente de determinación $R^{2}$ ajustado fue $=0.990$, por lo que $99 \%$ de la variación total en la demanda de carne de pollo en canal para la región CE se ha explicado mediante el modelo de regresión y representa un nivel satisfactorio de explicación del modelo como un todo

En otras palabras, a un nivel de confianza de $95 \%$, se rechaza la hipótesis de que cualquiera de las variables independientes no esté correlacionada con la demanda de carne de pollo en canal. Todas las variables predeterminadas fueron estadísticamente significativas; su respectiva $\mathrm{t}$-calculada fue superior a $2.00(\mathrm{P}<0.01)$. El estadístico Durbin-Watson (1.30) indicó la existencia de un nivel bajo de autocorrelación entre las series de tiempo. En tanto que el valor de la prueba Brusch-Pagan y White fue suficiente para confirmar homocedasticidad y no colinealidad, aunado a que el error estándar de cada estimador fue de magnitud insuficiente como para obtener un valor de t-calculada no significativa; por tanto, no hubo multicolinealidad en el modelo estimado.

\section{ANÁLISIS ECONÓMICO}

Con base en los resultados indicados en el cuadro 1, los signos aritméticos que anteceden a cada estimador (variable independiente) del modelo de demanda de carne de pollo en canal en la región CE de México resultaron acordes a lo indicado por la teoría económica. El modelo estimado es predictivo, tiene lógica económica y sirve para realizar proyecciones del comportamiento de la variable dependiente. La significancia estadística y los signos aritméticos adecuados de las variables predeterminadas son condiciones necesarias y suficientes para afirmar que el modelo también presenta significancia económica. En consecuencia, es fiable estimar las elasticidades respectivas (cuadro 2) y analizar el comportamiento de la demanda de carne de pollo en canal en esa región de México.

Con base en las condiciones establecidas en este trabajo, el comportamiento de la demanda de carne en 
canal de pollo en la región CE de México, durante el periodo 1996-2018, fue sensible a la dinámica regional tanto del precio del producto en cuestión, como del ingreso, del precio de la carne de bovino, del precio de la carne de cerdo y del comportamiento creciente de la población (cuadro 2).

Los modelos que se probaron consideraron los diversos trabajos realizados en la estimación de la demanda de cualquier producto (Ramírez-González et al. 2003; Vázquez y Martínez 2015; Cruz-Jiménez et al. 2016; Puebla et al. 2018). En los que algunos señalan que para modelos de demanda se sugiere ajustar los datos de variables predeterminadas a modelos multiplicativos (linealizados con logarítmicos), en lugar de modelos lineales multivariables, debido a que producen mejores resultados para explicar el comportamiento de la variable dependiente (Brigham y Pappas 1995). Sin embargo, en este estudio, el modelo exponencial sugerido por la literatura no produjo los mejores resultados. Incluso, los signos de los coeficientes de las variables fijas mostraron el signo contrario, de acuerdo con la teoría microeconómica, y no hubo significancia estadística individual: el error estándar multiplicado por dos superaba al valor de cada estimador.

En consecuencia, al contrastar esos resultados con los del modelo de regresión lineal múltiple (MRLM), los del segundo fueron congruentes con la teoría y todas las variables predeterminadas fueron estadísticamente significativas; por esas razones, se decidió la estimación del modelo de regresión lineal múltiple para explicar el comportamiento de la variable explicada (la demanda de carne de pollo en canal en la región CE de México).

Así, la demanda de carne de pollo en canal para la región de estudio cambia linealmente con las fluctuaciones de las variables independientes. El intercepto (cuadro 1) no representa algún significado económico, queda lejos del abanico de datos observados y no se puede concebir a la demanda de carne de pollo como negativa cuando las variables independientes consideran un valor de cero.

El coeficiente de cada variable explicativa indica la relación marginal entre dicha variable y la demanda de carne de pollo en canal (ceteris paribus). Por ello, la cifra -7.60, el coeficiente del PCPR - el precio real asignado a la carne de pollo en canal-, significa que la cantidad demandada de carne de pollo en canal en la región CE de México decrece casi ocho mil toneladas por cada incremento de un peso mexicano por kilogramo en el precio de la carne de pollo (ceteris paribus).
Por su parte, el coeficiente del PIBPR - la variable PIB per cápita (ingreso per cápita) - revela que por cada mil pesos mexicanos adicionales de ingreso per cápita disponible en favor del consumidor de esta carne, se da, en promedio, un aumento esperado de 0.86 miles de toneladas en la demanda de carne de pollo en canal.

En adición, la cifra 4.22, el coeficiente del precio de la carne de bovino, afirma que la demanda de carne de pollo en canal podría aumentar en 4,220 t debido al aumento de un peso mexicano por kilogramo en el precio de la carne en canal de bovino.

Con relación a la variable fija PRCC, su coeficiente de -5.99 expresa que por cada peso mexicano por kilogramo de aumento en el precio de la carne de cerdo en canal se espera (ceteris paribus) que la demanda de carne de pollo decrezca en 5.99 miles de t. Análogamente, 76.20, el valor del coeficiente asignado a la variable POB, expresa que la demanda de carne de pollo en la región de estudio podría incrementarse (ceteris paribus) en 76.20 miles de toneladas, por cada millón de incremento en el número de consumidores de la región CE de México. Tal fluctuación hará que la curva de la demanda del producto se desplace hacia la derecha y el consumo esperado de carne de pollo en canal aumente en 76,200 t.

Para el periodo considerado, la cantidad demandada de pollo, tuvo un comportamiento inverso e inelástico (-0.21) al precio del producto; este resultado fue cercano al -0.36 que obtuvo Bathi (1987) sobre demanda de carne de pollo en Australia. En otras palabras, la cantidad demandada de carne de pollo en canal en esa región de México fue inelástica a su precio. Este resultado es consistente con la afirmación de Tomek y Kaiser (2014), quienes mencionan que, por lo general, los productos básicos tienen a comportarse como inelásticos; consecuente, asimismo, con el de Vázquez y Martínez (2015), donde la elasticidad precio de la demanda de carne de pollo en México fue -0.47 (inelástica) (estimado mediante un sistema de demanda casi ideal); por su parte, el resultado se aleja del acierto de Ramírez-González et al. (2003), cuya elasticidad precio de la demanda de pollo fue elástica (-1.19), estimada mediante ecuaciones simultáneas; es cercano al de Cruz-Jiménez et al. (2016), cuyo valor fue -0.19 , estimado en huevo para plato en México, con ecuaciones simultáneas, y alejado pero consistente con el resultado de Rubí-González et al. (2018), cuya elasticidad fue -0.009 en su estudio de demanda de carne de pollo en la CDMX.

Es de esperarse que, ante incrementos porcentuales unitarios esperados en el precio, ceteris paribus, de la 
carne de pollo en canal para la región de estudio, la cantidad demandada de este cárnico disminuya en menos de $1 \%$, de ahí su inelasticidad al precio de adquisición de este subproducto pecuario. El valor de la elasticidad precio de la demanda de carne de pollo en canal es, relativamente, pequeño $(-0.21)$, dada la existencia de pocos sustitutos cercanos, como la carne de bovino; incluso, es inelástica, debido a que esta carne se considera como un bien necesario en la dieta de los mexicanos (Vázquez y Martínez 2015).

Con base en el resultado de la elasticidad ingreso (0.09) de la demanda, este producto se considera como un bien normal necesario e inelástico al ingreso (cuadro 2) (Leroy y Meiners 1990; Nicholson y Snyder 2015; Parkin y Loría 2015); por tanto, es de esperarse que, ante incrementos porcentuales unitarios en el ingreso (ceteris paribus) per cápita del consumidor de esa región, la demanda de carne de pollo en canal crezca en $0.09 \%$; resultado análogo a 0.98 (inelástica al ingreso) de Cruz-Jiménez et al. (2016), pero en huevo para plato; asimétrico con el de Ramírez-González et al. (2003), para el periodo 1970-1998; alejado de los hallazgos de González-Sánchez (2001), quien obtuvo una elasticidad ingreso en carne de pollo de 2.60, y de Rubí-González et al. (2018), quienes concluyeron una elasticidad ingreso de la demanda de carne de pollo en la CDMX de 1.08.

Por tanto, en las condiciones planteadas en esta investigación, por cada $1 \%$ de incremento en el ingreso real del consumidor, se espera que la curva de la demanda se desplace hacia la derecha y provoque un incremento de $900 \mathrm{t}$ de carne de pollo en canal, por lo que este determinante continuará siendo factor importante para incrementar el consumo de este producto en la región CE. Durante el periodo analizado, el ingreso real per cápita de esa región creció en promedio 0.09\% (CONAPO 2019; INEGI 2019), lo que impactó, ceteris paribus, en un crecimiento de la demanda de carne de pollo en canal, para el mismo periodo, de $5.60 \%$.

Por su parte, la elasticidad cruzada entre el precio de la carne de pollo y el de la carne de bovino fue mayor que cero (0.16); es decir, fue positiva, y con base en este resultado, estos dos bienes son sustitutos. Ese resultado significa que por cada $1 \%$ de aumento en el precio al consumidor de carne de pollo en la región CE de México, la cantidad demandada de esta carne tenderá a disminuir, en tanto que cabe esperar que la demanda por carne de bovino se incremente en $0.16 \%$. La aseveración anterior es acorde con lo que encontraron Ramírez-González et al. (2003) y Rubí-González et al. (2018), quienes afirmaron una elasticidad cruzada entre las carnes de pollo/bovino de 0.02 .

En este mismo sentido, el valor de la elasticidad pollo/cerdo fue de -0.16 , lo que clasifica a la carne de cerdo como un bien complementario de la de pollo en esta región. Tal resultado no contradice la teoría microeconómica, pero en las condiciones en las que se analiza la información - por ejemplo, periodos y regiones diferentes - no siempre es de esperarse que, en la demanda, los productos sustitutos mantengan ese comportamiento (es decir, como sustitutos). Así, es probable que cada $1 \%$ de disminución en el precio real de la carne de cerdo impacte en el crecimiento de la demanda por carne de pollo en esa región de estudio. Al respecto, durante el periodo de análisis, el precio real de la carne de cerdo decreció $0.46 \%$ (SIAP 2020) y la demanda de carne de pollo creció, en promedio, $5.6 \%$. Este resultado es opuesto con el de Ramírez-González et al. (2003), en su estudio para México, al confirmar una relación positiva (0.47) entre carne de pollo y carne de cerdo, y con el de Vázquez y Martínez (2015), cuya relación entre ambas carnes fue de 0.15; pero converge con el de Ramírez et al. (2011), al concluir un efectivo negativo de -0.006 para ambas carnes (bienes complementarios).

Con referencia a la población humana, la demanda de carne de pollo en canal fue elástica (2.46) al número de consumidores (POB), lo cual significa que por cada unidad porcentual en que se incremente el número de consumidores (POB), se espera que la demanda por carne de pollo en canal en esa región aumente $2.46 \%$ y la curva de la demanda se desplace hacia la derecha. De hecho, de 1996 a 2018, la población en la región CE creció en promedio 1.29\%, situación que, ceteris paribus, hizo acrecentar la demanda por carne de pollo 5.6\%. El hallazgo anterior converge con el de Rubí-González et al. (2018), cuya elasticidad de la población para la CDMX fue de 3.07. 
Cuadro 1. Resultados de la salida del modelo de demanda de carne en canal de pollo en la región CE de México, 1996-2018

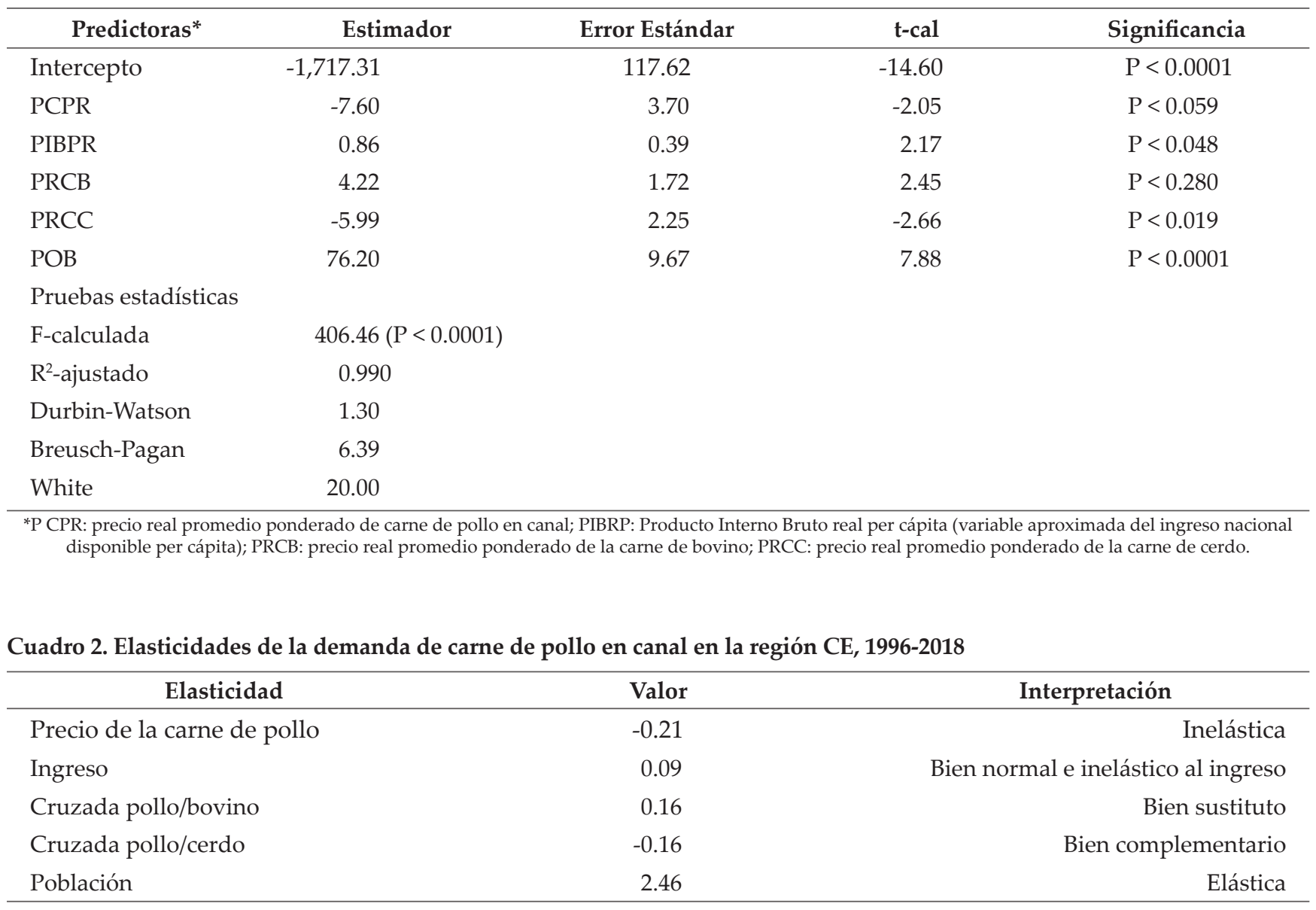

\section{Conclusiones}

Contrario a la teoría, el modelo estadístico que mejor ajustó la masa de datos fue el de regresión lineal múltiple. Así, durante el periodo de análisis se confirmó que las bondades de ese modelo y sus conclusiones sobre la realidad en la actividad avícola ratifican que la demanda de carne de pollo en canal en la región CE de México actuó de forma elástica a la población e inelástica al nivel de ingreso real per cápita, al verse más influenciada por tales variables, clasificándose como un bien normal e inelástico al ingreso. La cantidad demandada de pollo tuvo una respuesta inversa e inelástica al precio real al consumidor de este producto; en tanto que la carne de cerdo actuó como un bien complementario a la demanda de pollo en la región de estudio. La demanda por carne de pollo continuará al alza debido al efecto positivo del ingreso real, al comportamiento de la población regional, así como al precio real de la carne de bovino y cerdo, y al precio real de la carne de pollo.

\section{Agradecimientos}

Los coautores agradecen a los estudiantes del Programa de Doctorado en Ciencias Agropecuarias y Recursos Naturales por la obtención de alguna parte de la información para la elaboración de los modelos utilizados. 


\section{LITERATURA CITADA}

Bassols BA. 1992. México: formación de regiones económicas. Primera reimpresión. Instituto de Investigaciones Económicas. UNAM. México, D. F.

Bathi UN. 1987. Supply and demand for poultry meat in Australia. Australian Journal of Agricultural Economics 31(3): 256-265. https://doi.org/10.1111/j.1467-8489.1987. tb00468.x.

Brigham EF, Pappas JL. 1995. Economía y Administración. Tercera edición. McGraw-Hill. México, D. F.

[CONAPO] Consejo Nacional de Población [internet]. 2019. Indicadores demográficos de México de 1950 a 2050 y de las entidades federativas de 1970 a 2050 [cited 2020 Mar 02]. Disponible en: https://datos.gob. $\mathrm{mx} /$ herramientas/indicadores-demograficos-de-mexico-de-1950-a-2050-y-de-las-entidades-federativas-de-1970-a-2050?category=web\&tag=economia.

Cruz-Jiménez S, García-Mata R, Mora-Flores JS, García-Sánchez RC. 2016. El mercado de huevo para plato en México, 1960-2012. Agricultura, Sociedad y Desarrollo 13(3): 385-399.

González-Sánchez, RF. 2001. Estimación de elasticidades de la demanda para la carne de res, pollo, cerdo y huevo en México, una aplicación del sistema de demanda casi ideal. Tesis de Doctorado. División de Ciencias Económico Administrativas. Universidad Autónoma Chapingo. Chapingo, Estado de México. México.

Gujarati DN, Porter DC. 2010. Econometría. Quinta edición. McGraw-Hill Interamericana. México, D. F.

[INEGI] Instituto Nacional de Estadística, Geografía e Informática [internet]. 2018. Banco de Información Económica. Índice Nacional de Precios al Consumidor [cited 2020 Mar 14]. Disponible en: https://www.inegi. org.mx/app/tabulados/default.aspx?nc=ca57_2018.

[INEGI] Instituto Nacional de Estadística, Geografía e Informática [internet]. 2019. Banco de Información Económica [cited 2020 Mar 01]. Disponible en: http:// www.inegi.org.mx/sistemas/bie/.

[FIRA] Fideicomisos Instituidos en Relación a la Agricultura [internet]. 2019. Panorama Agroalimentario. Carne de pollo 2019 [cited 2020 Mar 01]. Disponible en: https://www.inforural.com.mx/wp-content/ uploads/2019/09/Panorama-Agroalimentario-Carne-de-pollo-2019.pdf.

Leroy R, Meiners RE. 1990. Microeconomía. Tercera edición. McGraw-Hill. México, D. F.

Medina JC, Rejón MJ, Valencia ER. 2012. Análisis de rentabilidad de la producción y venta de pollo en canal en el municipio de Acanceh, Yucatán. Revista Mexicana de Agronegocios 16(30): 909-919. https://doi. org/10.22004/ag.econ.120499.

Nicholson W, Snyder C. 2015. Teoría Microeconómica. Principios básicos y ampliaciones. Décimo primera edición. Cengage Learning. México, D. F.

Parkin M, Loría E. 2015. Microeconomía, un enfoque para América Latina. Décima edición. Pearson Educación. México, D. F.
Puebla S, Rebollar S, Gómez G, Hernández J, Guzmán E. 2018. Factores determinantes de la oferta regional de carne bovina en México, 1994-2013. Región y Sociedad 30(72): 1-17. https://doi.org/10.22198/rys.2018.72.a895.

Ramírez-González A, García-Mata R, García-Delgado G, Matus-Gardea JA. 2003. Un modelo de ecuaciones simultáneas para el mercado de la carne de pollo en México, 1970-1998. Agrociencia 37(1): 73-84.

Ramírez TJ, Martínez MA, García R, Hernández A, Flores JS. 2011. Aplicación de un sistema de demanda casi ideal (AIDS) a cortes de carne de bovino, porcino, po1lo, huevo y tortilla en el periodo de 1995-2008. Revista Mexicana de Ciencias Pecuarias 2(1): 39-51.

Rebollar-Rebollar E, Rebollar-Rebollar S, Gómez-Tenorio G, Guzmán-Soria E. 2019. Efecto de las importaciones en la oferta regional de carne de pollo en México, 1996-2016. Tropical and Subtropical Agroecosystems 22: 415-425.

Rubí-González Y, Rebollar A, Rebollar S, Rebollar E, Hernández R. 2018. Modelo econométrico de demanda de carne de pollo en la Ciudad de México, 1996. Revista Mexicana de Agronegocios 43(22): 99-106.

[SAGARPA] Secretaría de Agricultura, Ganadería, Desarrollo Rural, Pesca y Alimentación [internet]. 2019. Escenario Base 2009-2018. Proyecciones para el sector agropecuario de México [cited 2020 Mar 02]. Disponible en: http://www.ruralfinanceandinvestment.org/ sites/default/files/Proyecciones $\% 20$ para $\% 20 \mathrm{el} \% 20$ sector\%20agropecuario\%20de\%20M\%C3\%A9xico.pdf.

Salvatore D. 2009. Microeconomía. Cuarta. edición. McGraw-Hill. México, D. F.

[SAS] Statistical Analysis System. 2003. SAS, Versión 9.1.3. Institute Inc, Cary. N.C., USA.

[SIAP] Sistema de Información Agroalimentaria y Pesquera [internet]. 2020. Anuario Estadístico de la Producción Ganadera [cited 2020 Mar 01]. Disponible en: https://nube.siap.gob.mx/cierre_pecuario/.

[SNIIM] Sistema Nacional de Información e Integración de Mercados [internet]. 2019. Mercados nacionales pecuarios. Pollo entero [cited 2020 Mar 01]. Disponible en: http://www.economia-sniim.gob.mx/nuevo/ Home.aspx?opcion=/SNIIM-Pecuarios-Nacionales/ MenAve.asp.

Tellez R, Mora JS, Martínez MA. 2016. Caracterización del consumidor de carne de pollo en la zona metropolitana del Valle de México. Estudios Sociales 48(26): 193209.

Tomek WG, Kaiser HM. 2014. Agricultural Product Prices. Cornell University Press. Ithaca. Nueva York.

[UNA] Unión Nacional de Avicultores [internet]. 2019. Panorama de la avicultura [cited 2020 Mar 01]. Disponible en: http://una.org.mx/industria/.

Vázquez JMP, Martínez MA. 2015. Estimación empírica de elasticidades de oferta y demanda. Revista Mexicana de Ciencias Agrícolas 6(5): 955-965.

Wooldridge JM. 2009. Introducción a la econometría. Un enfoque moderno. Cuarta edición. Cengage Learning. México, D. F. 